\title{
Tomada de decisão da criança e família: visão da enfermagem
}

\author{
Mariana Lucas da Rocha Cunha ${ }^{1}$
}

\section{Resumo}

A sociedade é frequentemente desafiada por transformações constantes que afetam os relacionalmentos humanos. Em nossa realidade assistencial lidamos com indivíduos de diferentes gerações, sejam com colegas de profissão, famílias ou os nossos próprios pacientes e é esperado que isto interfira nas relações que estabelecemos com eles. O ambiente de cuidado também vem se transformando, influenciado pelas transições da economia e pelos avanços tecnologicos. A enfermagem dentro deste contexto evoluiu de atores puramente assistencialista para gestores do cuidado, do ambiente e dos recursos. E as transformações deste cenário vem influenciando o cuidado às crianças e famílias, sobretudo, no que se refere a cuidado compartilhado entre família e equipe. A tomada de decisão da criança e família no contexto hospitalar vem sendo construida ao longo do tempo, entre conflitos e expectativas pouco objetivas do que se espera de cada elementos no cenário do cuidado. A possibilidade de estabelecer uma relação colaborativa entre criança-família e a equipe de enfermagem configura-se como elemento para promoção da autonomia e consequente tomada de decisão compartilhada entre os envolvidos neste processo.

Palavras chave: Tomada de decisão; criança hospitalizada; família; cuidado em enfermagem.

Cunha, Mariana Lucas da Rocha. "Tomada de decisão da criança e família: visão da enfermagem", in Anais do 2‥ Congresso Internacional Sabará de Especialidades Pediátricas

\footnotetext{
${ }^{1}$ Enfermeira. Doutora em Enfermagem pela Escola de Enfermagem da Universidade de São Paulo (EEUSP). Docente da disciplina de Enfermagem Pediátrica e Oncológica na Graduação em Enfermagem da Faculdade Israelita de Ciências da Saúde Albert Einstein (FICASE). Coordenadora da Pós-Graduação em Enfermagem Pediátrica e Neonatal da FICSAE. Mariana.cunha@eisntein.br.
} 
A sociedade é frequentemente desafiada por transformações constantes que afetam os relacionalmentos humanos. Em nossa realidade assistencial lidamos com indivíduos de diferentes gerações, sejam com colegas de profissão, famílias ou os nossos próprios pacientes e esperado que isto interfira nas relações que estabelecemos com eles.

Nos deparamos com indivíduos nascidos em diferentes gerações que apresentam crenças, comportamentos e necessidades condizentes com a sua época. Para aqueles indivíduos conhecidos como Geração X ou "Perdida”, nascidos entre 1961-1979, são considerados “descolados” ou modernos, gostam de tecnologia e procuram-se comportar e ter uma aparência jovial. São focados e interessados. ${ }^{(15)}$

Pessoas pertencentes a Geração Y ou “nativos digitais”, são definidos como a geração da internet, nascidos nos meados de 1970 até meados de 1990, nasceram em um mundo de avanços tecnológicos e prosperidade economica. Muitas vezes, foram filhos de pais carentes de atenção, e desta maneira, foram superestimados com presentes, atenções e atividades, são percebidos como impacientes. ${ }^{(15)}$

Na denonimada Geração Z ou “Zapping”, nascidos na segunda metade da década de 1990 e nos anos de 2000, são elementos muito familiarizados com o "webworld”, têm como características trocar o tempo todo o foco de atenção nas coisas (como televisão, telefone, internet). São voláteis na profissão, para eles, a comunicação não tem barreiras e são imediatistas, impacientes e impetuosos. ${ }^{(15)}$

O ambiente de cuidado também vem se transformando, influenciado pelas transições da economia e pelos avanços tecnologicos. Os hospitais em boa parte da nossa realidade, perdeu o foco das casas de irmandades, que tinham como objetivos primordiais prestar caridade e abrigar pobres e desabrigados, principalmente quando doentes ou por morrer ${ }^{(12)}$, sem se preocupar com o resultado financeiro. As constantes descobertas na área da saúde durante o século XX, o desenvolvimento da tecnologia, a crescente sofisticação da hotelaria, a necessidade de expansão de serviços de apoio à assistência e outros fatores, fizeram com que a organização hospitalar, gradualmente, se tornasse mais complexa, com características e objetivos diferentes. ${ }^{(12)}$ Atualmente, são considerados como grandes empresas, que além do cuidado, precisam se preocupar com o retorno do investimento, modernização e otimização de recursos materiais e humanos.

A enfermagem dentro deste contexto também evoluiu de atores puramente assistencialista para gestores do cuidado, do ambiente e dos recursos. E a ética do cuidado em enfermagem, seguindo paralelamente as transformações da sociedade, também sofreu grandes mudanças, originariamente de uma postura de subserviência médica, que progrediu lentamente para a acompreensão da autonomia profissional e que atualmente vem enfrentando complexos desafios frente aos padrões éticos e legais que têm se desenvolvido nos direitos do consumidor, do paciente e dos avanços tecnológicos. Fez-se 
necessário definir o que significa uma boa prática de enfermagem, embasada nos princípios da ética como autonomia, beneficência, não maleficência e justiça. ${ }^{(5)}$

O conceito de promoção da autonomia embasa a condição primária para a tomada de decisão. A tomada de decisão é um processo que começa antes e termina depois da decisão em si. Dentro do contexto de saúde, pacientes devem ser livres para escolher ou rejeitar as intervenções nos cuidados de sua saúde, sem coerção, e devem ser dadas todas as informações necessárias sobre sua condição e os tratamentos possíveis, para que eles possam fazer uma escolha informada. ${ }^{(5)}$ É preciso incluir a família nesta questão quando tratamos da criança doente e hospitalizada.

A tomada de decisão envolve três elementos principais nesta realidade de cuidado em saúde: o hospital (ou ambiente de cuidado), a criança e sua família e a equipe de saúde. O impacto que cada elemento tem no processo de decisão necessita ser delineado e neste sentido, iremos focalizar uma fração da equipe de saúde, de fundamental importância que é a enfermagem.

O hospital é percebido como um ambiente complexo e despersonalizado, regulado por ações mecanizadas, com regras e rotinas específicas, burocrátizado e com a cultura própria de cada serviço. $(8,17)$ Existem vários hospitais em um mesmo hospital, o contexto da unidade de terapia intensiva difere do ambulatório, o ambiente da unidade de internação não se compara ao do centro cirúrgico, além de que, muitas vezes, a criança e a família precisam lidar com a internação em unidades de adultos. Essas situações demandam da criança e família adaptação constante nas suas relações com a equipe de saúde, no seu comportamento e no atendimento de suas necessidades.

A família frente a doença e hospitalização da criança apresenta reações e sentimentos decorrentes desta experiência, influenciados por perceções e emoções alteradas, que afetam sua capacidade de compreender a situação. ${ }^{(14)}$ Normalmente a vivência da família no hospital é caracterizada por um período de desestruturação familiar, realinhamento de papeis, surgimento de sentimentos como incerteza e ameaça a sua integridade, além de medo, tristeza e ansiedade. ${ }^{(7)} \mathrm{A}$ depender da gravidade da doença e o fato da hospitalização ser um evento estressantemente inesperado, submete a família a dependência da equipe de saúde, descontrole, fragilidade e isolamento social; que deflagram ameaça a sua autonomia e consequente estado de vulnerabilidade. ${ }^{(6)}$

Frente as normas e rotinas hospitalares, o familiar pode sentir sua vida invadida pela imposição dos deveres institucionais diversos a suas crenças, valores e habitos de vida e seu contexto social/familiar. ${ }^{(18)}$ Há momentos em que a família aceita a situação imposta, concordando com as regras estabelecidas ou resignando-se às condições, e outros enfrenta as dificuldades, buscando seus direitos, tendo confiança e esperança, desejando participar do tratamento, querendo ser conhecida pela equipe e contando com a rede de apoio. ${ }^{(6)}$

As relações da família com a equipe de saúde e os conflitos decorrentes destes relacionamentos; além dos aspectos que envolvem a participação da família no cuidado da criança doente e internada, vem sendo explorado de maneira substancial na assistência pediatrica. ${ }^{(10)}$ 
A compreensão desta perspectiva nos obriga a considerar que na história, o envolvimento da família pela enfermagem, se deu de modo inverso, ou seja, a enfermagem teve seu início nos domicílios dos pacientes, o envolvimento familiar era natural. A transição das prática de enfermagem para o contexto hospitalar , durante a Segunda Guerra Mundial, decorreu no afastamento das famílias dos cuidados de seus membros doentes e de grandes eventos no ciclo da vida como nascimento e morte. Com a evolução dos estudos e da assistência propriamente dita, a enfermagem iniciou um novo ciclo no cuidado às famílias, com ênfase e a obrigação de convidar as famílias para participar novamente de seus próprios cuidados de saúde. ${ }^{(19)}$

Na realidade brasileira, a inclusão da família neste cenário, iniciou com a preocupação de humanizar o cuidado a criança enferma, entendida como uma situação de crise, ao tira-la do seu cotidiano e a colocando em contato com uma realidade desconhecida. A questão da humanização hospitalar começou a ser discutida durante os anos 80, com o "Programa Mãe Participante" e consolidou-se nos anos 90, com o chamado "Família Participante”. Estes programas visavam minimizar o sofrimento psíquico das crianças e fortalecer a capacidade de resposta favorável ao tratamento proposto. ${ }^{(14)}$

O marco conceitual da inserção da família, foi sobretudo, a regulamentação do Estatuto da Criança e do Adolescente, que garante a permanência de um acompanhante em período integral durante a hospitalização infantil. ${ }^{(3)}$ No entanto, sabe-se que a inserção da família exigia das organizações de saúde, adaptação nos processos de trabalho e realinhamento da relações interpessoais, que na prática, não aconteceu e fomentou as relações conflituosas entre família e equipe de enfermagem. ${ }^{(16)}$

Com a inserção da família no ambiente hospitalar, o foco do cuidado da enfermagem ampliou para o binômio criança-família. No entanto, como este processo não foi embasado por um planejamento gradativo, mas, sobretudo adaptado a concepção individual dos profissionais quanto ao que significava compartilhar o cuidado da criança, a família foi inserida com a expectativa de realizar cuidados às crianças rotulados pela equipe como simples ou "semelhantes aos domiciares”, ainda que ações de responsabilidade da enfermagem. ${ }^{(16)}$

A equipe de enfermagem tem realizado um cuidado centrado em tarefas e a família tem sido incluida como uma realizadora de cuidados. Há o reconhecimento de que o trabalho da enfermagem está se distanciando do cuidado integral e humanizado, e portanto, as necessidades do binômio criança e família não têm sido identificados nas suas singularidades pelos enfermeiros. ${ }^{(16)}$

Contudo, estudo revela que apesar de parecer satisfatório, de algum modo, a dinâmica do cuidado à família estabelecida pelos enfermeiros pediátricos, os mesmos vivem dilemas éticos nesta realidade. Onde os enfermeiros sentem-se angustiados na tentativa de proteger os direitos dos pacientes, preocupam-se com a falta de informações oferecidas a criança e família o que afeta diretamente a autonomia do binômio, enfrentam grandes desafios na rotina de trabalho com condições pouco favoráveis de trabalho e dificuldades de comunicação entre a equipe interdisciplinar. ${ }^{(5)}$ 
Parte da solução para estes dilemas, é considerar algumas premissas para o dia-a-dia de trabalho da enfermeira com as famílias, é acreditar que "a família tem um impacto significativo sobre a saúde e o bem-estar de cada um de seus membros, podendo exercer considerável influência sobre suas enfermidades”, “ a doença é um assunto de família” e ainda que, “ enfermeiras têm o compromisso, bem como a obrigação ética e moral, de envolver as famílias em seus cuidados de saúde.” ${ }^{19}$ ) Cada uma destas assertivas, carrega em si, o olhar para a família como um sistema e que o que afeta um dos indivíduos afeta aos outros também. ${ }^{(2,19)}$

Para a enfermeira é alinhando o planejamento e a implementação da sua assistência aos principios do Cuidado Centrado na Família (CCF), que são: Considerar a família uma constante na vida dos indivíduos, acreditar que o cuidado deve ser construido com base na colaboração entre os envolvidos, que há necessidade da informação ser compartilhada entre os envolvidos (criança-família e a equipe de saúde), que há de se trazer valores e crenças de ambos os lados desta relação, reconhecer que cada indivíduo tem sua maneira de enfrentamento, favorecer a existência de uma rede social de apoio e finalmente, que as famílias frente às situações de crises como doença ou hospitalização são famílias ${ }^{(4)}$, que se pode favorecer a autonomia da criança e família.

A enfermeira que trabalha com crianças e família tem múltiplos papéis, dependendo das necessidades dessas, das situações e da assistência, deve desempenhar o papel de "Educadora de saúde”, onde ensina formal ou informalmente as famílias sobre saúde e enfermidade e, age como principal provedor de informações de saúde; "Prestadora de cuidados de saúde”, prestando cuidados diretos bem como supervisão dos cuidados prestados por outros; "Conselheira da família”, onde deve ter um papel terapêutico para ajudar a enfrentar problemas e identificar recursos; "Defensora da família”, trabalhando no sentido de dar apoio às famílias. Estes papéis servem como base para as intervenções com as famílias e para a promoção do relacionamento positivo entre os envolvidos. ${ }^{(19)}$

O relacionamento entre a enfermeira e a família é parte essencial do cuidado da criança e família ${ }^{(17)}$, a qualidade e a natureza dos relacionamentos influenciam na experiência de cuidado tanto para a família quanto para a enfermeira. As relações colaborativas entre as enfermeira e as famílias devem ser pautadas em cinco pressupostos: ${ }^{(19)}$

1. O relacionamento família-enfermeira caracteriza-se pela reciprocidade.

2. O relacionamento família-enfermeira não é hierárquico.

3. Enfermeiras e famílias têm, respectivamente, habilidades especializadas para manter a saúde e lidar com os problemas de saúde.

4. Enfermeiras e famílias trazem pontos fortes e recursos ao relacionamento famíliaenfermeira.

5. Processos de feed-back podem ocorrer de modo simultâneo em níveis diferentes do relacionamento.

Estabelecer relações colaborativas é parte essencial no contexto da tomada de decisão da criança e família. Para que a tomada de decisão seja competente deve envolver o conhecimento dos 
riscos e das consequências de cada opção de escolha ${ }^{(11)}$, onde os papeis que devem ser incorporadas pelas enfermeiras podem auxiliar no fornecer informações, defender os direitos da criança e família e ter um papel terapêutico.

Para as crianças, especificamente, as decisões de saúde devem ser baseadas no que é melhor para a criança, não no que é melhor para os outros. O padrão de melhor interesse é importante em pediatria, pois ajuda a proteger as crianças. Deve-se considerar o equilíbrio entre benefícios e potenciais danos da decisão a ser tomada e se possível pelo melhor juiz desta decisão. Outra questão importante é solicitar o assentimento da criança para os procedimentos que são possíveis. “Assentimento implica que a informação de saúde deve ser prestada às crianças, adaptada à sua capacidade de compreender, e sua cooperação voluntária deve ser solicitada tanto quanto for razoavelmente possível.” (5)

Em estudo realizado para analisar as situações e contextos que propiciaram ou dificultaram as relações de acolhimento e autonomia, em uma enfermaria pediátrica, demonstrou que o princípio da autonomia foi respeitado nos momentos de cuidados das mães em relação aos seus filhos, nas conversas com as equipes e na apropiação do seu espaço e do processo de tratamento. ${ }^{(1)}$ Este estudo reforça a importância da autonomia das famílias, nos momentos que para a enfermagem, muitas vezes podem ser considerados inexpressivos.

Frente ao exposto, fica evidente que as famílias podem vivenciar relações positivas no hospital quando se sentem potencializadas para desenvolver o cuidado à criança e conseguem desenvolver o dialogo com a equipe de enfermagem, ${ }^{(8)}$ vivenciando uma relação colaborativa. É a prática do cuidado compartilhado, preconizado por ações humanísticas e ações de cuidados na interação dialógica com a criança e a família ${ }^{(10)}$, que permea a autonomia e consequente a decisão compartilhada.

O cuidado compartilhado com a criança e família deve abranger, entre outros requisitos: respeitar a criança e família e inclui-las no plano terapêutico ( respeitar a individualidade), valorizar a participação da família no cuidado da criança (definir papéis e o que se espera de cada um), instituir praticas cuidativas embasadas no dialogo, na negociacao e na participação, sensibilizar e instrumentalizar os profissionais de saúde e sobretudo, ter apoio das instituições onde o cuidado é realizado. ${ }^{(16,18)}$

As transformações promovidas pela realidade que estamos enfrentando no cenário do cuidado em saúde a criança e a família, indicam que as mudanças no papel do enfermeiro se fazem necessárias, quando percebemos que incluir a criança e família na tomada de decisão, pode favorecer a recuperação da criança, melhorar a satisfação da criança e família, possivelmente diminuir o tempo de internação e consequentemente os custos do cuidado em saúde. 


\title{
Child and family decision making: the nurse's view
}

\author{
Mariana Lucas da Rocha Cunha ${ }^{1}$
}

\begin{abstract}
Society is often challenged by the constant transformations affecting human relationships. In delivering care, we deal with individuals from different generations. These individuals can be coworkers, relatives or even our patients. To some extent, generational differences are expected to interfere with the relationships we establish with other people. The care environment has also been transformed by economic transitions and technological advances. In this context of change, nurses have progressed from strictly care professionals to managers of the care environment and resources. This transformation has influenced the delivery of care to children and families, especially with regard to shared care between family and health team. Decision making among children and their families in the hospital context has been constructed over time, but this process has been the source of conflict. In addition, the expectations of each individual in the care scenario have been unclear. Establishing a collaborative relationship between the child and his or her family and the nursing team helps promote autonomy and, consequently, shared decision making among those involved in this care process.
\end{abstract}

Keywords: Decision Making; Hospitalized Child; Family Nursing; Pediatric Nursing;

\section{Referencias}

1. Alves CA; Deslandes SF; Mitre RMA. Desafios da humanização no contexto do cuidado da enfermagem pediátrica de média e alta complexidade. Comunicação Saúde Educação. 2009; 13(1): 581-94.

2. Bousso RS. A teoria dos sistemas familiares como referencial para as pesquisas com famílias que experienciam a doença e a morte. Rev Min Enferm. 2008 Abr-Jun;12(2): 257-61.

3. Brasil. Ministério da Saúde. Estatuto da Criança e do Adolescente Brasília. 1991.

4. Callery P; Smith L A. Study of Role Negotiation Between Nurses and the Parents of Hospitalized Children. Journal of Advanced Nursing 1991; 16: 772-781

5. Carnevale F. Considerações éticas em Enfermagem Pediátrica. Rev. Soc. Bras. Enferm. Ped. 2012. 12(1): 37-47. 
6. Côa TF; Pettengill MAM. A experiência de vulnerabilidade da família da criança hospitalizada em Unidade de Cuidados Intensivos Pediátricos. Rev Esc Enferm USP. 2011; 45(4):825-32.

7. Cunha MLR. Incerteza e sacrifício: o sofrimento na vida familiar invadida pelo câncer da criança. Tese de Doutorado. 2009. Escola de Enfermagem da Universidade de São Paulo.

8. Gomes GC; Nicola GDO; Souza NZ, Chagas MCS, Farias DFR; Xavier DM. Percepções da família acerca das dificuldades de adaptação da criança à hospitalização: subsídios para a enfermagem. Cogitare Enferm. 2013; 18(4): 767-74.

9. Gomes GC; Erdmann AL; Oliveira PK; Xavier DM; Santos SSC; Farias DHR. A família durante a internação hospitalar da criança: contribuições para a enfermagem. Esc Anna Nery 2014;18(2):234-240.

10. Lima AS; Silva VKBA; Collet N; Reichert APS; Oliveira BRG. Relações estabelecidas pela enfermeiras com a família durante a hospitalização infantil. Texto Contexto Enferm. 2010; 19(4): 700-08.

11. Martinho MGCM, Martins MMPFS, Angelo M. Escala de conflito em tomadas de decisão em saúde: instrumento adaptado e validado para língua portuguesa. Rev Esc Enferm USP 2013; 47(3):576-83).

12. Melo IM. Humanização da assistência hospitalar no Brasil: conhecimentos básicos para estudantes e profissionais. (Acesso em : Setembro de 2014.) Disponível em http://www.hcnet.usp.br/humaniza/pdf/livro/livro_dra_inaia_Humanizacao_nos_Hospitais _do_Brasil.pdf

13. Murakami R; Campos CJG. Importância da relação interpessoal do enfermeiro com a família de crianças hospitalizadas. Rev Bras Enferm. 2011; 64(2): 254-60.

14. Oliveira FMF; Constantino CF; Barros JCR; Hirschheimer MR. In: Constantino CF; Barros JCR; Hirschheimer MR. Cuidando de crianças e adolescentes sobr o olhar da ética e bioética. São Paulo: Atheneu, 2009. Cap 11: Humanização no Atendimento pediatrico. 173-87.

15. Pereira N. Seja um professor nota 10. Rio de Janeiro: Ed Livre Expressão. 2011. p. 205.

16. Pimenta EAG; Collet N. Dimensão cuidadora da enfermagem e da família na assistência à criança hospitalizada: concepções da enfermagem. Rev Esc Enferm USP. 2009; 43(3): 622-9.

17. Robinson C. Health care relationships revisited. Journal of family nursing. 1996; 2(2)133151.

18. Xavier DM; Calcagno GC; Santos SSC; Lunardi VL; Pintanel AC; Erdmann AL. A família na Unidade de Pediatria: convivendo com normas e rotinas hospitalares. Rev Bras Enferm. 2014; 67(2): 181-6. 
19. Wright LM; Leahey M. Enfermeiras e Famílias. Guia para avaliação e intervenção na família. 5ª edição. São Paulo: Ed. Roca. 2012. Cap1: 1-20. 